\& J. Edu. \& Sci., Vol. (21), No. (4) 2008 \&

\title{
The Influence Of Hole Radius On The Properties Of Photonic Crystal Fiber
}

\section{Huda Masood Mohamad \& Abdulghafoor Ibrahim Abdullah laser student2@yahoo.com \\ ghaa4@yahoo.com}

\section{Department of Physics / College of Education University of Mosul}

Received

$10 / 06 / 2007$
Accepted

15 / 08 / 2007

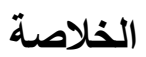

مة البهث في تأثير نصف قطر الفجوة الهوائية على كل من الفتحة العددية والتشتت الكلي

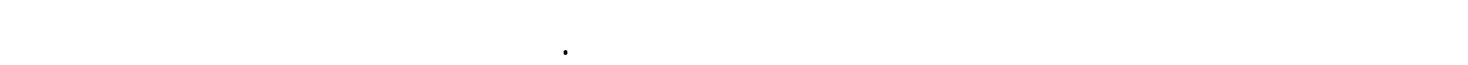

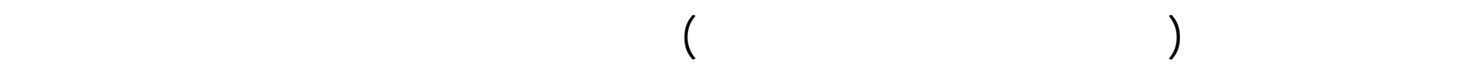

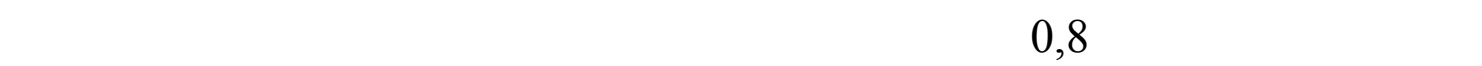

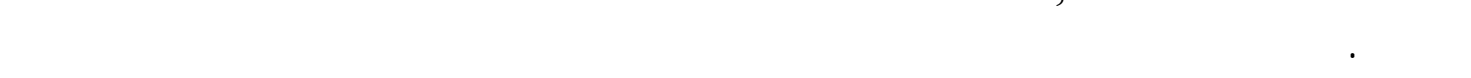
الزياةة في نصف قطر الفجوة. اللف البلوري الفوتوني ذو التركيب 0,8 مايكرون وعند موجة إسب لـ 1.0 مـ ايكرون

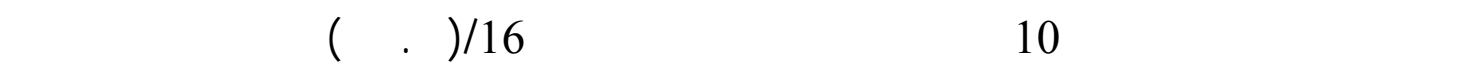

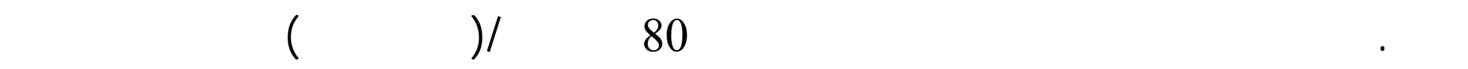

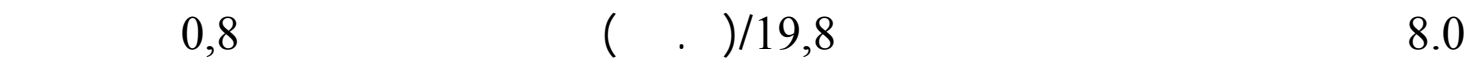
الليزرالنيتانيوه - الياقوت الأزرق.

\section{Abstract}

The influence of air hole radius on the numerical aperture, total dispersion, effective area, and nonlinear coefficient for the photonic crystal fibers are investigated. This study shows, when the pitch size and the number of rings holes are held constant, the increasing of air hole radius up to $0.8 \mu \mathrm{m}$ gives better coupling efficiency due to the increasing in numerical aperture. The results show that the zero dispersion point is shifted toward the shorter wavelength due to the increasing of air hole radius. 
The PCF structure of $0.8 \mu \mathrm{m}$ gives an effective area $10 \mu \mathrm{m}^{2}$, nonlinear coefficient $16 \mathrm{~km}^{-1} \mathrm{~W}^{-1}$ and zero dispersion at communication wavelength $1.0 \mu \mathrm{m}$. While the same structure gives negative dispersion 80 $\mathrm{ps} /(\mathrm{nm} . \mathrm{km})$, effective area $8 \mu \mathrm{m}^{2}$ and nonlinear coefficient $19.8 \mathrm{~km}^{-1} \mathrm{~W}^{-1}$ at communication wavelength $0.8 \mu \mathrm{m}$ of Ti-Za laser.

\section{1- Introduction}

Photonic crystal fibers (PCFs) or microstructured optical fibers (MOFs) were proposed in 1992. The first working example of PCF was made by Knight et al in 1996 [1]. In comparison with conventional optical fibers (COFs), guided propagation of the electromagnetic field in the PCFs is achieved by the introduction of defects. These defects form a periodic pattern of air holes around a silica core, and acts as a cladding of the PCF[2]. The effective refractive index is considered as an average over the solid silica and the air holes it contains. Thus the cladding region has a lower average refractive index than the core and guides light similar to a step index optical fiber, as shown in Fig.1 [2].

Conventional step-index fiber Photonic crystal fiber
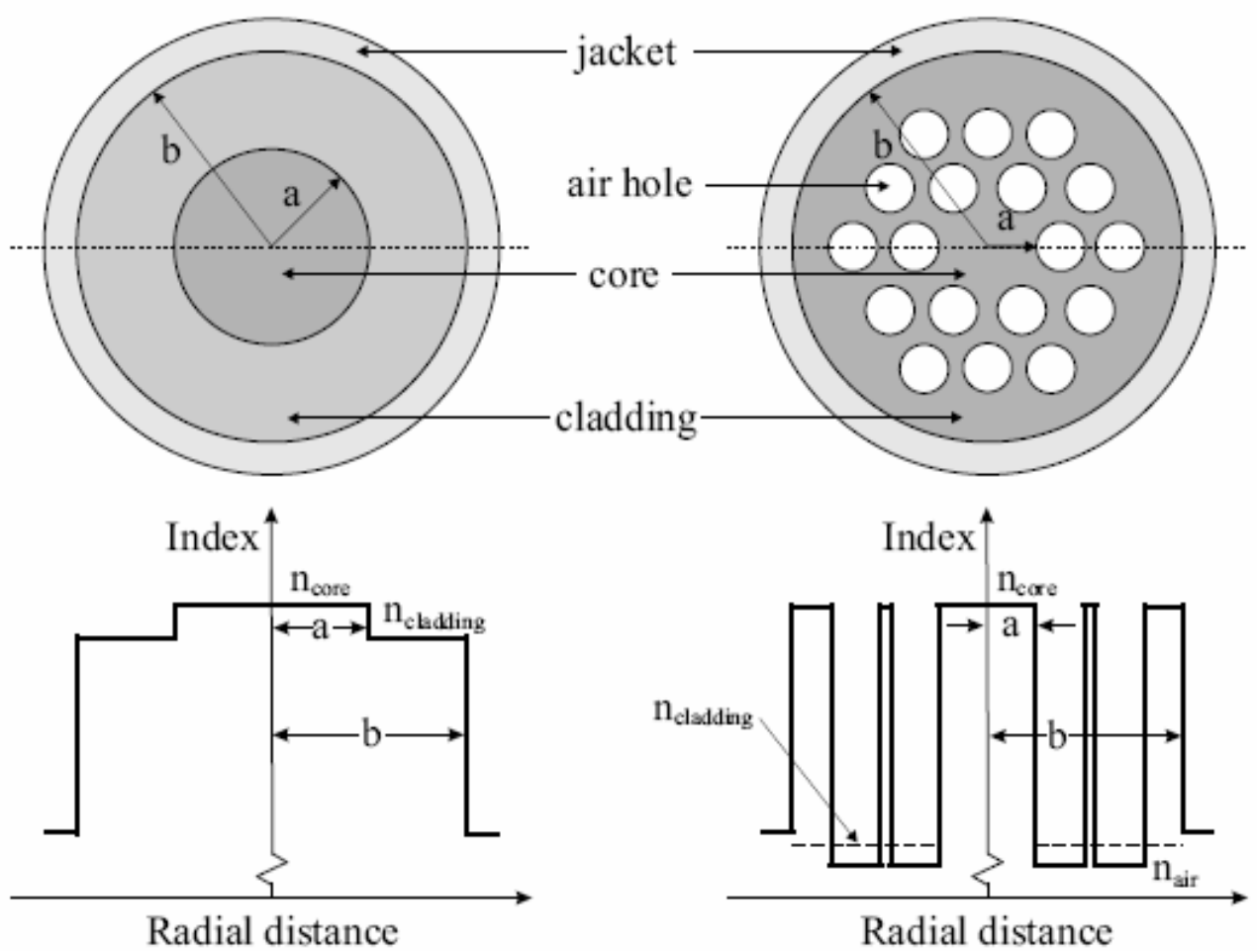

Fig.1: Schematic illustration of the cross section and refractive index profile for a step index optical fiber and index guiding photonic crystal fiber [2]. 
The basic structure of the PCF is shown in Fig.2 [3], the small circles of diameter $\mathbf{d}$ represents air holes that run the length of the fiber, and $\boldsymbol{\Lambda}$ is the distance from two centers of holes, and called the pitch size. The dashed circle identities the location of the core, which may be either solid or air, depending on the design and intended purpose. In either case, the region represents a defect in the structural pattern. If solid, the core radius extends to the inner edge of the first ring of holes, that means the air holes are typically arranged in a triangular lattice. The silica core guiding the light is formed by the missing air hole. The guidance mechanism depends on the nature of the defects and the air hole arrangement [3].

According to the guidance mechanism, PCF can be divided in to two categories:[4] total internal reflection (TIR), where air holes surround a high index solid core, and photonic band gap (PBG), where the light is confined to a central air hole $[4,5]$

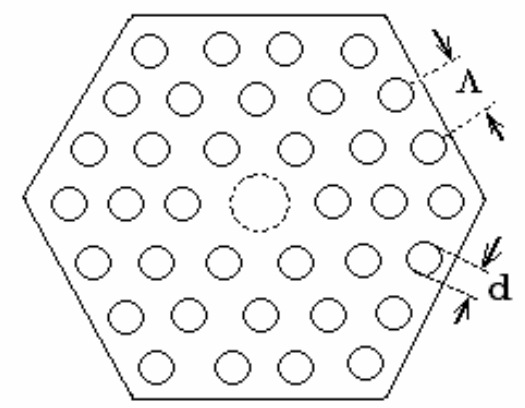

Fig. 2: Basic structure form of photonic crystal fiber [3].

PCFs are found to exhibit remarkable properties which include single mode and the nearly-zero dispersion (NZD) [6], flattened dispersion, and ultra-flattened dispersion over a wide range of the electromagnetic spectrum [6-8], that means, achieving good control of dispersion.

In practical applications however only those characteristics mentioned above may not be enough for justifying the usefulness of the PCF. The numerical aperture, dispersion parameters, effective area, nonlinear coefficient, and nonlinear length are needed for some particular applications. Such characteristics and its applications will be the aim of this work.

This paper is organized as follows: in section 2, the theory, we introduce the theoretical relations and their definitions such as: numerical aperture, normalized frequency nonlinear effect which are related to the PCF. In section 3 we report the results and discussion for PCFs. Finally conclusions and future work are given in section 4.

\section{2- Theory}


PCFs have several unique optical properties: high numerical aperture single mode over wide range of wavelength, flattened or ultra flattened dispersion over a wide range of wavelength. These are because the refractive index contrast between the core $n_{c}$ and the refractive index of the surrounding region $n_{c l}$ is large and the effective refractive index $n_{\text {eff }}$ of the surrounding region is strongly dependent on wavelength. These properties are determined by selecting the appropriate arrangement of air hole diameter, pitch size, and the number of rings. The theoretical relations and their definitions are described as the following.

\subsection{Numerical Aperture NA}

It is an important characteristics of an optical fiber showing its gathering ability of the incident light over wide range of angles, for PCF the $\mathrm{NA}$ is given as the following [2]

$$
\mathrm{NA}=\sin \theta_{\mathrm{NA}}=\sqrt{\mathrm{n}_{\mathrm{c}}^{2}(\lambda)-\mathrm{n}_{\mathrm{eff}}^{2}(\lambda)}
$$

Where $\theta_{\mathrm{NA}}$ is the half divergence angle.

Low NA indicates a small acceptance angle, so coupling to low NA fiber is more difficult and less efficient than coupling to high NA, also NA relates to the effective area $A_{\text {eff }}$; for Gaussian field of width $w$, or spot size, one has the standard approximate expression, $\tan \theta=\lambda / \pi w$, for the half divergence angle $\theta$ of the light radiated from the end-facet of the fiber. The relation of the NA in terms of $A_{\text {eff }}$ which was used in a study by Mortensen [9] and Mortensen et al [10] to study the numerical aperture of PCF is:

$$
A_{\text {eff }}=\frac{\lambda^{2}}{\pi}\left(\frac{1}{\mathrm{NA}^{2}}-1\right)
$$

The effective area is a quantity of a great importance because it is a measure of nonlinearities in optical fibers, and can be used to enhance or prevent the nonlinear effect, depending on the value of $A_{\text {eff. }}$. In case of $w>\lambda$, Mortensen et al [10] showed that $A_{\text {eff }}=\pi w^{2}$.

\subsection{Normalized Frequency $V$}

The value of $\mathrm{V}$ determines the number of confined modes to be exist in the core. In the context of PCF, Wong [2] and Mortensen et al [10] made a modification to that equation which is used in standard step index fiber[3 ].

If $n_{\text {clad }}$ is replaced by $n_{\text {eff }}$ and a ( the core radius) by $\Lambda$ to get:

$$
\mathrm{V}=2 \pi \frac{\Lambda}{\lambda} \sqrt{\mathrm{n}_{\mathrm{c}}^{2}(\lambda)-\mathrm{n}_{\text {eff }}^{2}(\lambda)}
$$

here $\Lambda, n_{c}, n_{\text {eff }}$ are the pitch size, refractive index of the core and the 
effective refractive index of the air hole array $[2,11]$. V- value in PCF stays fixed value even if the wavelength shortened, because of the strong wavelength dependence of the $n_{\text {eff }}$. The special aspect of PCF is that with decreasing wavelength, the effective cladding index also increase, this serving the offset the increase in V-value. This means that PCF can offer single mode operation from the visible to infrared region, as shown in Fig.3 [12], such a feature is not possible with conventional single mode fiber, where $\mathrm{V}$-value rises as the wavelength shortens.

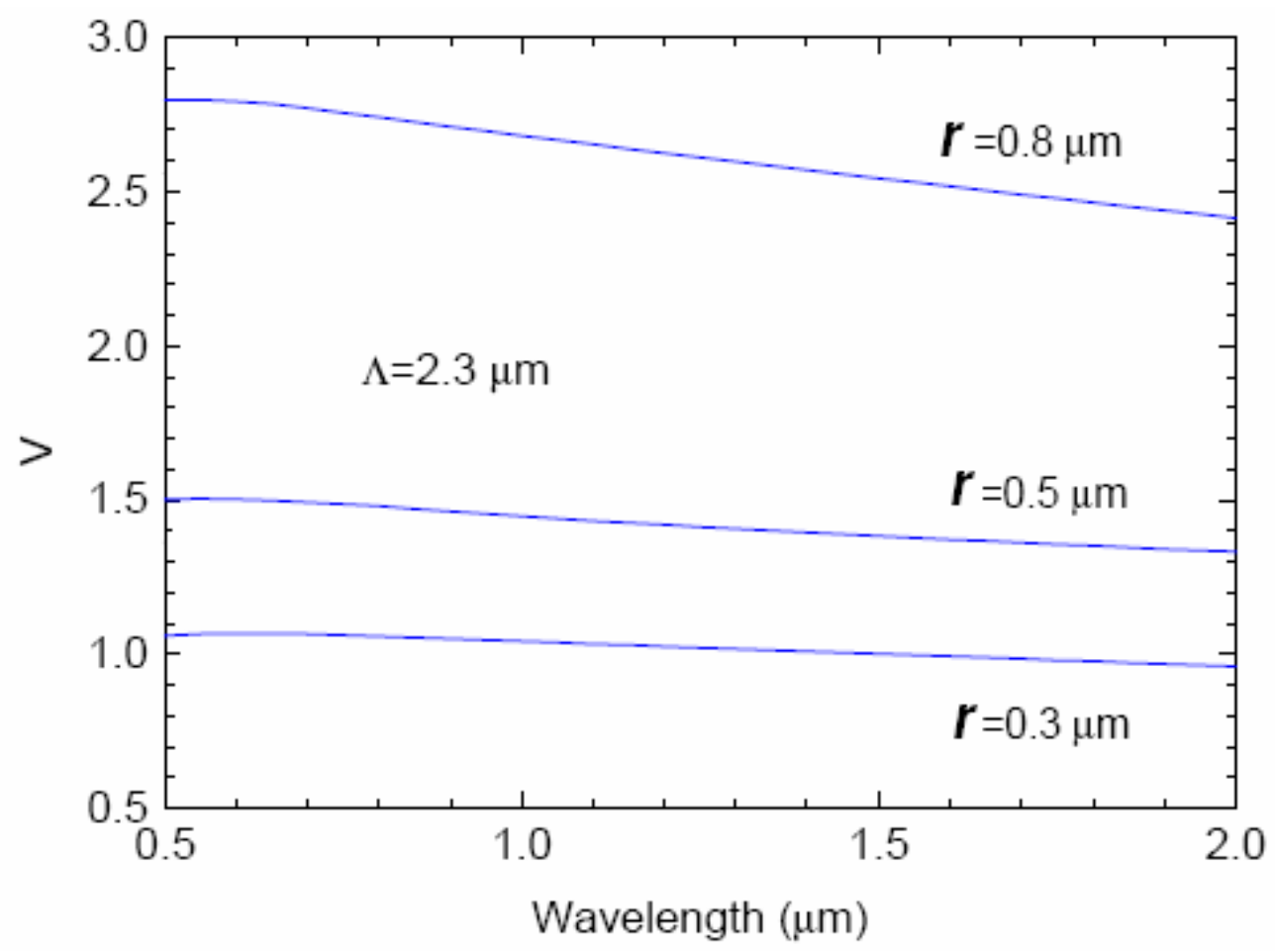

Fig. 3: Calculated parameter V vs. wavelength for PCFs with different airhole radius[12].

\subsection{Nonlinear Effect in PCF}

The nonlinear optical effects in dielectric media studied by Franken et al $[13,14]$. When they observed the frequency doubling of ruby laser beam by quartz crystal, this was soon followed by the observation of a plethora of other nonlinear phenomena including third harmonic generation, optical rectification, parametric amplification and oscillation, self focusing and self phase modulation. Because optical nonlinearities are intrinsically weak (at least far from material resonance), these developments depend critically on 
the availability of high power laser (peak power $>\mathrm{kW}$ ) and small effective area of the optical fiber [13].

Knowledge of $A_{\text {eff }}$ is thus an important starting point in the understanding of nonlinear phenomena. The high index contrast between silica and air, the PCF technology offers the possibility of much tighter mode confinement and therefore a lower effective area compared to standard fiber[8].

One can say, that the different nonlinear effect in fiber can be either useful or undesirable depending on the application [3]. In modern communication systems which involves high power level and employ optical amplifiers, the understand and use of nonlinear effect is extremely important.

A- Nonlinear coefficient $\gamma$ :

The nonlinear coefficient $\gamma$ in terms of $A_{\text {eff }}$ is given by $[9,13]$

$$
\gamma=2 \pi \frac{\mathrm{n}_{2}}{\lambda A_{\text {eff }}}
$$

where $\mathrm{n}_{2}$ is nonlinear - index coefficient in the nonlinear part of the refractive index of silica fiber and equal to $2.5 \times 10^{-20} \mathrm{~m}^{2} \mathrm{~W}^{-1}$

B- Dispersion length parameters $L_{D}$ :

This parameter can be interpreted as a distance over which the effects of quadratic dispesion $\beta_{2}$ become significant, and given by the following relations[3]:

$$
\mathrm{L}_{\mathrm{D}}=\frac{\mathrm{T}^{2}}{\left|\beta_{2}\right|}
$$

Where $\mathrm{T}$, is the pulse width.

The effects of fiber dispersion $\beta_{2}$ can be understood by expanding the mode propagation constant $\beta(\omega)$ in a Taylor series about the carrier frequency $\omega_{0}$ as $[2,3]$ :

$\beta(\omega)=\beta\left(\omega_{0}\right)+\left(\omega-\omega_{0}\right) \beta_{1}+\left(\frac{1}{2 !}\right)\left(\omega-\omega_{0}\right)^{2} \beta_{2}+\left(\frac{1}{3 !}\right)\left(\omega-\omega_{0}\right)^{3} \beta_{3}+\ldots \ldots$

Where $\beta_{m}=\left(\frac{\partial^{\mathrm{m}} \beta}{\partial \omega^{\mathrm{m}}}\right), \mathrm{m}=0,1,2$,

$\beta_{2}$ is group velocity dispersion :

$$
\beta_{2}=\frac{1}{c}\left(2 \frac{d n}{d \omega}+\omega \frac{d^{2} n}{d \omega^{2}}\right)
$$


Recently many photonic crystal fibers operate in zero or nearly zero dispersion that means $\beta_{2}$ equal or approaches zero, which means terms of high order in Eq. 6 may be important [2, 3, 7].

C- Nonlinear length $L_{N L}$ :

This parameter is defined in term of $A_{\text {eff }}$ as the following [3]

$$
L_{N L}=\frac{c \cdot A_{e f f}}{\omega_{0} \cdot n_{2} \cdot p_{0}}
$$

Where $\mathrm{c}, \omega_{0}$ and $p_{0}$ are light velocity, angular frequency and the power of the laser light transmitted via optical fiber respectively. Knowledge of $L_{D}$ and $L_{N L}$ one can determine the soliton order $N$ or the relative strength of the nonlinearity:

$$
N \equiv\left(\frac{L_{D}}{L_{N L}}\right)^{\frac{1}{2}}
$$

where $N$ in Eq. 9 is a variable parameter, provides a measure of the relative strengths of the nonlinearity and the dispersion [3].

\section{3- Results and Discussion}

The published data of Zhu Z. and Brown T. G. [12] have been implemented in our calculations for total dispersion and other properties of PCFs for the fundamental mode. The effective index of different PCFs obtained by [12] are based on full vectorial method. Such a method is simple and efficient approach, which overcomes the general limitations of the plane wave expansion.

Three PCFs of same pitch size $(\Lambda=2.3 \mu \mathrm{m})$ and different hole radius $(\mathrm{r}=0.3,0.5,0.8 \mu \mathrm{m})$, without missing hole in the center of the core, are considered. These fibers can be single mode over very large spectral range $(0.5 \mu \mathrm{m}$ to $2.0 \mu \mathrm{m})$ as shown in Fig.(3). The material dispersion of silica is considered in the calculation [12], using Sellmier equation with the parameters for fused silica as given in Ref. [14].

\section{3-1 Numerical Aperture NA( $\lambda)$}

Fig. 4 shows the influence of the air hole radius on the numerical aperture. The results show that by increasing the wavelength, the numerical aperture increased, one can say by adjusting the air hole radius, a high freedom in designing a fiber with a certain NA at a desired wavelength [10]. The high NA, (0.25 in this work, 0.3 [1] and 0.9 [15] depends on the 
structure of the PCF) at wavelength $1.55 \mu \mathrm{m}$, make the coupling between different PCFs easy and allowing very fast beams to propagate down the fiber. Such a property is not possible in conventional single mode fiber.

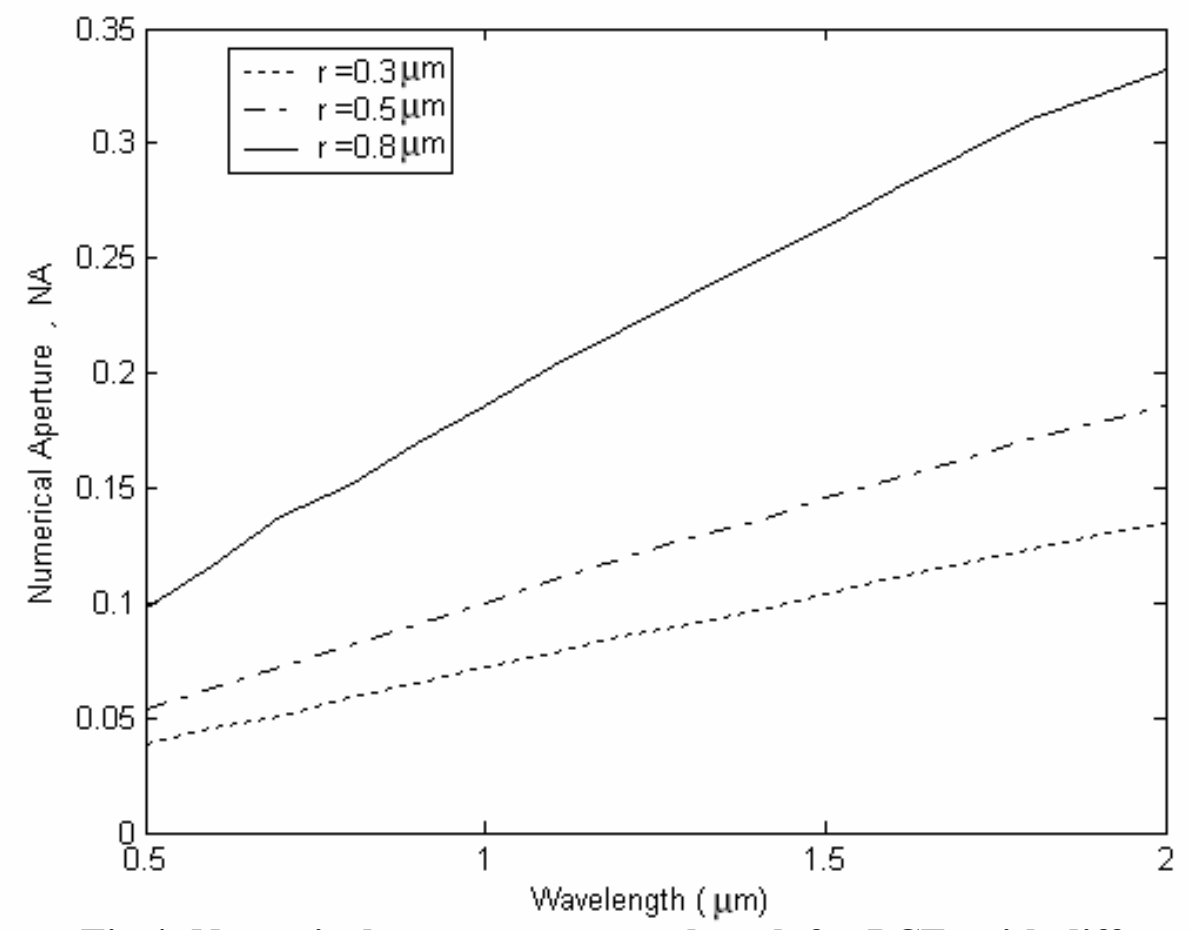

Fig.4: Numerical aperture vs. wavelength for PCFs with different air- hole Radius.

\section{3-2 Dispersion $D(\lambda)$}

The dispersion was calculated as a function of wavelength, using the definition $\left(D(\lambda)=(-\lambda / c) d^{2} n_{e f f} d \lambda^{2}\right)$ [2,3], for different air hole radius and is illustrated as in Fig.(5). From this figure it is noted that for small air radius $(\mathrm{r}=0.3 \mu \mathrm{m})$ the dispersion curve is close to the material dispersion of pure silica, where the zero dispersion wavelength $\lambda$ ZD around $1.3 \mu \mathrm{m} \mathrm{[16].} \mathrm{The}$ figure shows also, the continuous increment of the air hole radius cause the $\lambda$ ZD point to shift towards the shorter wavelength $[17,18]$ and down shifts the dispersion to negative value dispersion $\sim 80 \mathrm{ps} /(\mathrm{km} . \mathrm{nm}$ ) (anomalous dispersion for $\lambda<\lambda_{\mathrm{ZD}}$ at $0.8 \mu \mathrm{m}$ Ti-Za laser) [7], while the fiber remain single mode. The large tailor ability in the design of the crystal fiber with respect to air hole radius provides further fruitful mean of tuning of dispersion curve to obtain specific properties.

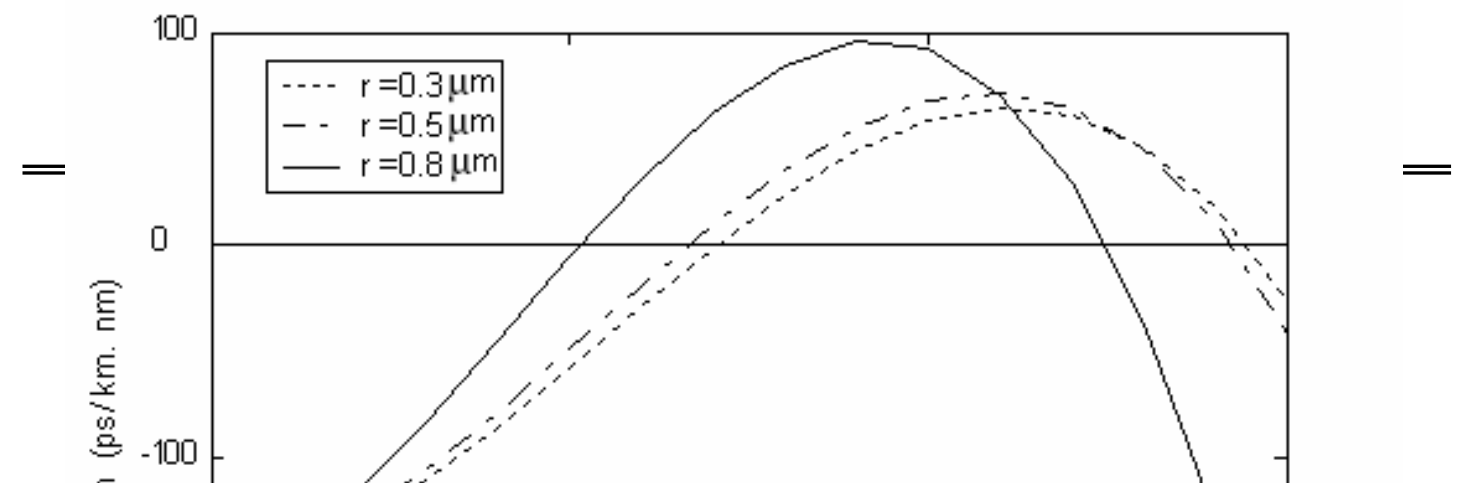


Fig.5: Dispersion computed vs. wavelength for PCFs with different air-hole radius.

\section{3-3 Effective Area Aeff( $\lambda)$ and Nonlinear Coefficient $\gamma(\lambda)$}

We have shown already that the different PCFs are exhibiting zero dispersion characteristics in different wavelengths see Fig.5. In practical applications however only the zero or near zero dispersion characteristics may not be enough for justifying the usefulness of the PCFs. The size of the effective area and the value of the nonlinear coefficient (as shown in Fig.6 and Fig.7 respectively) both are a function of wavelength, which are strongly related to each other to determine their functions in linear or nonlinear application. The results of this work shows that the proposed PCF structure of $\mathrm{r}=0.8 \mu \mathrm{m}$ has in addition both the above mentioned characteristics which are small effective area $\sim 8 \mu \mathrm{m}^{2}$ and large nonlinear coefficient $\sim 20 \mathrm{~km}^{-1} \mathrm{~W}^{-1}$ at Ti-Za laser window $(\lambda=0.8 \mu \mathrm{m})$. While the PCF of structure $\mathrm{r}=0.3 \mu \mathrm{m}$ gives larger effective area $\sim 58 \mu \mathrm{m}^{2}$ and smaller nonlinear coefficient $\sim 3.0$ $\mathrm{km}^{-1} \mathrm{~W}^{-1}$ at the telecommunication wavelength of $\lambda=0.8 \mu \mathrm{m}$, Ti-Za laser. These PCFs with such properties in addition with high $\mathrm{NA}(\lambda) \sim 0.13$ has been experimentally verified to result in high coupling efficiencies between single mode fiber and nonlinear PCF of small effective area [19].

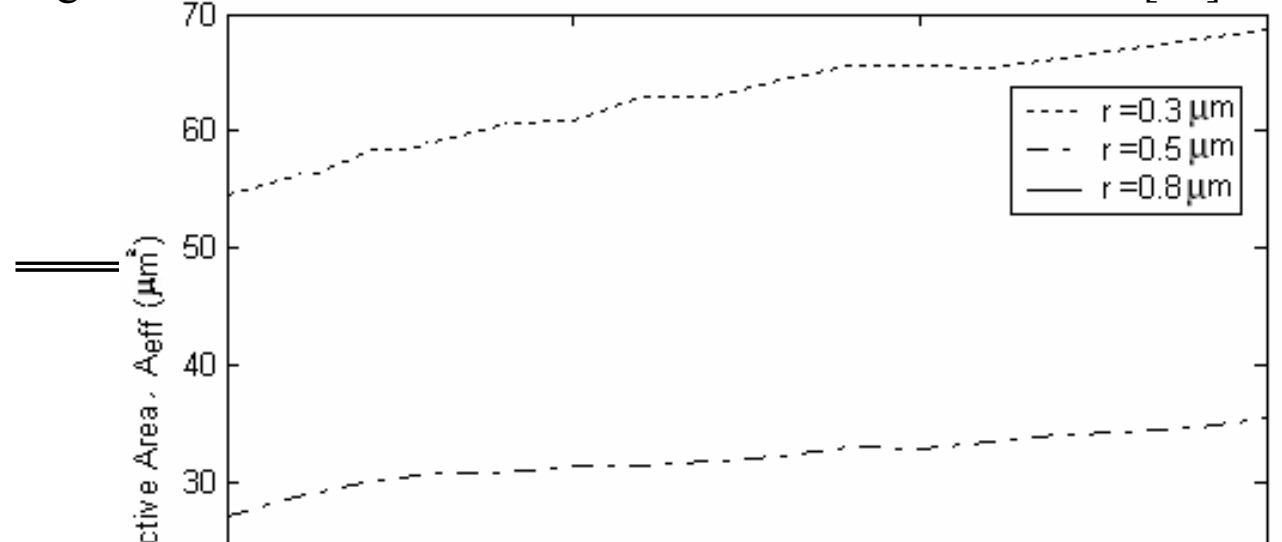


Fig.6: Effective area vs. wavelength for PCFs with different air-hole radius.

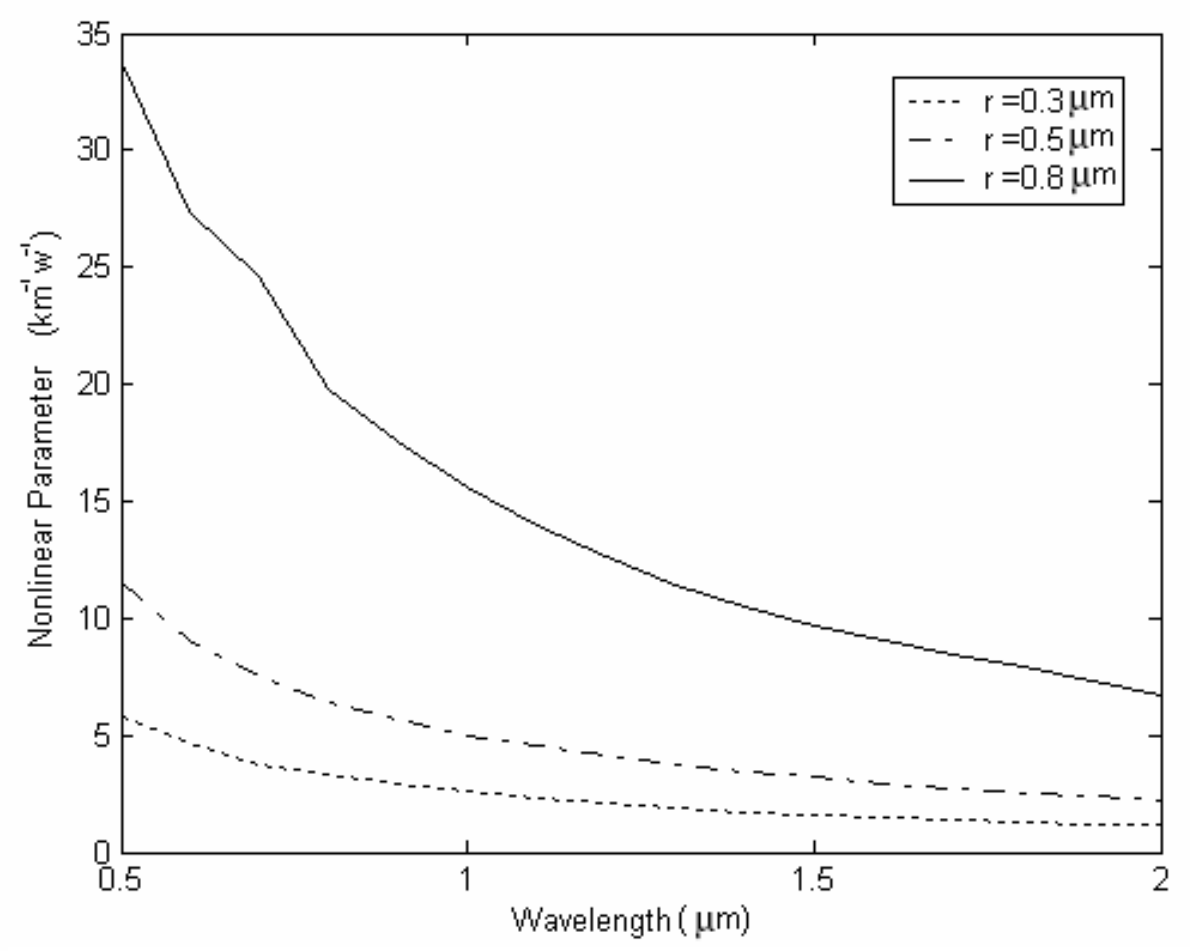

Fig. 7: Nonlinear parameter vs. wavelength for PCFs with different air-hole 


\section{3-4 Soliton Order $N$}

The application of the proposed structure of (PCF of $0.8 \mu \mathrm{m})$ is in soliton formation. Soliton are observed at different wavelengths; $0.85 \mu \mathrm{m}$ [20] and $1.5 \mu \mathrm{m}$ [12], and these solitons are possible when chromatic dispersion is negative, which for conventional silica fiber occur at wavelength above $1.3 \mu \mathrm{m}$ [21], while in case of PCF, negative dispersion depends strongly on the structure of the PCF. In this paper, using Eq.7, PCF of structure $0.8 \mu \mathrm{m}$ gives negative chromatic dispersion around $242 \mathrm{psec}^{2} / \mathrm{km}$ at the telecommunication wavelength of $0.8 \mu \mathrm{m}$ of Ti-Za laser.

When the peak intensity is $1.145 \times 10^{7} \mathrm{~W} / \mathrm{cm}^{2}$, the input pulse $T_{F W H M}=176.27 \mathrm{ps}$ long (the pulse duration $\left.T_{0}=T_{F W H M} /(2 \ln (1+\mathrm{V} 2))\right)[13]$, and according to Eqs.5,8,9 and when $A$ eff $=8.73 \mu \mathrm{m}^{2}$ the nonlinear length and the soliton order will be $44.4 \mathrm{~m}$ and 30.5 respectively such a structure has a high nonlinearity.

In comparison with other published work, when the $T_{F W H M}=200 \mathrm{fs}$ long, the intensity equal to $1.832 \times 10^{7} \mathrm{~W} / \mathrm{cm}^{2}, A$ eff $=7.5 \mu \mathrm{m}^{2}$ the soliton order is 19 at wavelength $1.5 \mu \mathrm{m}$ [13]. The results indicate that the proposed structure of a PCF has relatively high nonlinearity.

\section{4- Conclusions}

In conclusion, we find that the influence of air hole radius has a significant effect on the PCF parameters. The increase in hole radius pushed the $\lambda_{\mathrm{ZD}}$ toward the shorter wavelength. Additionally the proposed PCF of $\mathrm{r}=0.8 \mu \mathrm{m}$ shows large negative dispersion $\sim 80 \mathrm{ps} /(\mathrm{km} . \mathrm{nm})$, small effective area $\sim 8 \mu \mathrm{m}^{2}$ and large nonlinear coefficient $\sim 20 \mathrm{~km}^{-1} \mathrm{~W}^{-1}$ at the telecommunication wavelength of $\lambda=0.8 \mu \mathrm{m}$. Ti-Za laser.

By adjusting the air hole size, the pitch size, and the number of the rings one can design flattened or ultra flattened PCF with specific properties, this will be the future work. 


\section{References}

1. Knight J.C., Birks T. A., Russell P. St. J., and Atkin D.M., Opt. Lett, 21:1547-1549, (1997).

2. Wong K. L. G., M.Sc. Thesis, University of Auckland (2003).

3. Buck J. A., "Fundamentals of optical fibers" $2^{\text {nd }}$ Ed. John Wiley \& Sons. Inc., Hoboken New Jersey (2004).

4. Cheng H., Crutchfield W. Y., Doerg M. and Greengard L., Opti. Exp., 12:3791-3805 (2004).

5. Reichenbach K. L. and Xu C., Opt. Exp., 13:3799-3806( 2005).

6. Wu T. L., and Chao C. H., J. of Light wave Tech., IEEE, 23:2055-2061, (2005).

7. Saitoh K., Florous N., and Koshiba M., "Opt. Exp., 13:21-26, (2005).

8. Saitoh K., Koshiba M., Hasegawa T. and Sasaoka E., Opt. Exp., 11:843-852, (2003).

9. Mortensen N. A Opt. Exp., 10:341-348, (2002).

10. Mortensen N. A. , Folkenberg J. R., Skovgaard P. M.W. and Broeng J. IEEE Photonic Technology Letters: preprint, http://arxiv.org/abs/physics/0202073 (2002).

11. Kawanishi, S., Yamamoto T., Kubota, H., Tanaka M. and Yamaguchi S. I., Mat. Res. Soc. Symp. Proc., 7969(W7.2.1- W7.2.11), (2004).

12. Zhu Z. and Brown T. G. Opt. Exp., 8:547-554, ( 2001).

13. Hilligsoe K. M., Progress report for Ph.D student, Dept. of Phys. And Astronomy, University of Aarhus, (2003).

14. Basch E. E. B., 'Optical Fiber Transmission", Howard W. Sams \& Co. (1986).

15. Haynes R., Bland-Hawthorn J., Large M. C. J., Klein K.F., and Nelson. G. rh@aao.gov.au: www.aao.gov.au.

16. Broeng J., Barou S. E., Bjarklev A., Sondergaard T. and Knudsen. E., DOPS-NYT2- 22-28(2000), ib@com.dtu.dk.

17. $\mathrm{Yu}$ C. P., and H.C.. "Research on photonic crystal fibers using finite difference electromagnetic analysis", f86025@ew.ee.ntu.edu.tw.

18. Kuhlmey B. T., White T. P., Renversez G., Maystre D. Botten L.C. C. Sterek M. and McPhedran R.C., J. Opt. Soc. Am.B.19:2331-2340 (2002).

19. Saitoh K., Florous, N. and Koshiba, M., Opt. Exp., 13:21- 26 (2005).

20. Wadsworth W.J., Knight J.C., Ortigosa-Blanch A., Mangan B. J., and Russell P.St.J., 1999, Optical Society of America, e-mail: w.j.wadsworth@bath.ac.uk

21. Einarsson G., "Principles of lightwave communications", John Wiley and Sons, England, (1996). 